\title{
Standard Model updates and new physics analysis with the Unitarity Triangle fit
}

\section{A.J. Bevan, M. Bona*}

Queen Mary, University of London

E-mail: a.j.bevaneqmul.ac.uk, m.bonaeqmul.ac.uk

M. Ciuchini

INFN, Sezione di Roma Tre

E-mail: Ciuchinieroma3.infn.it

D. Derkach

LAL-IN2P3 Orsay

E-mail: derkach@lal.in2p3.fr

E. Franco, L. Silvestrini

INFN, Sezione di Roma

E-mail: enrico.franco@roma1.infn.it, Luca.Silvestrini@romal.infn.it

\section{Lubicz, C. Tarantino}

INFN, Sezione di Roma Tre, and Università di Roma Tre

E-mail: Iubicz@fis.uniroma3.it, tarantinodfis.uniroma3.it

\section{G. Martinelli}

INFN, Sezione di Roma and Università di Roma "La Sapienza"

E-mail: Guido.Martinelli@romal.infn.it

\section{F. Parodi, C. Schiavi}

Università di Genova and INFN

E-mail: fabrizio.parodi@cern.ch, Carlo.Schiavi@ge.infn.it

\section{Pierini}

CERN

E-mail: maurizio.pieriniecern.ch

\section{Sordini}

IPNL-IN2P3 Lyon

E-mail: Viola.Sordinidcern.ch

\section{A. Stocchi}

IN2P3-CNRS et Université de Paris-Sud

E-mail: achille.stocchi@lal.in2p3.fr

\section{Vagnoni}

INFN, Sezione di Bologna

E-mail: vincenzo.vagnoni@bo.infn.it 
We present the update of the Unitarity Triangle (UT) analysis performed by the UIfit Collaboration within the Standard Model (SM) and beyond. Within the SM, combining the direct measurements on sides and angles, the UT turns out to be over-constrained in a consistent way, with some tension due to recently included contributions to the theoretical prediction of $\varepsilon_{K}$ and the updated lattice average for $B_{K}$. Generalising the UT analysis to investigate NP effects, constraints on $b \rightarrow s$ transitions are also included and both CKM and NP parameters are fitted simultaneously. The most interesting results on the $b \rightarrow s$ transitions come from the $B_{s}-\bar{B}_{s}$ mixing and the di-muon charge asymmetry with updated results recently available from the Tevatron experiments. We observe a departure from the $\mathrm{SM}$ in the $B_{s}$ sector.

Flavour Physics and CP Violation - FPCP 2010

May 25-29, 2010

Turin, Italy

* Speaker. 

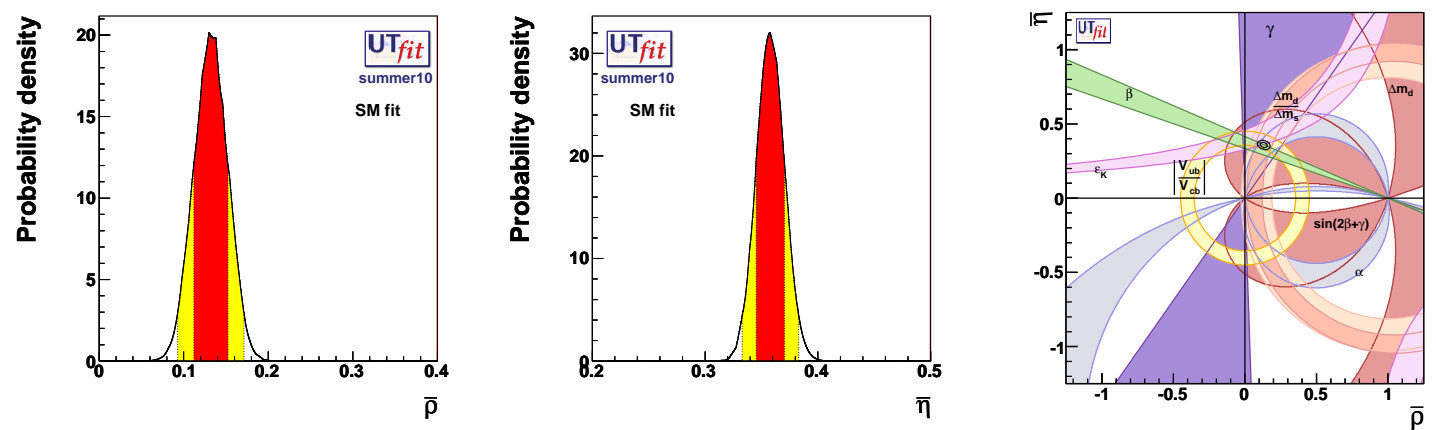

Figure 1: Result of the UT fit within the SM. Left and middle: 1D distributions for $\bar{\rho}$ and $\bar{\eta}$ resulting from the full SM fit. Right: the $\bar{\rho}-\bar{\eta}$ plane. The black contours display the $68 \%$ and $95 \%$ probability regions selected by the fit. The $95 \%$ probability regions selected by the single constraints are also shown with various colours for the different constraints.

\section{Standard Model Unitarity Triangle Analysis}

We present an update of the Unitarity Triangle (UT) analysis performed by the UTfit Collaboration following the method described in refs. [1], 目].

We use the latest determinations of the theoretical and experimental parameters. The basic constraints are $\left|V_{u b} / V_{c b}\right|$ from semileptonic $B$ decays, $\Delta m_{d}$ and $\Delta m_{s}$ from $B_{d, s}^{0}$ oscillations, $\varepsilon_{K}$ from $K$ mixing, $\alpha$ from charmless hadronic $B$ decays, $\gamma$ and $2 \beta+\gamma$ from charm hadronic $B$ decays, and $\sin 2 \beta$ from $B^{0} \rightarrow J / \psi K^{0}$ decays [3]. On the theoretical side, the non-perturbative QCD parameters are taken from the recent lattice QCD determinations: for $K$ parameters we refer to [ $₫$ ], for $B$ parameters to [5] and finally for exclusive $V_{u b}$ and beyond the SM $B$ parameters to [6]. The complete set of numerical values used as inputs can be found at the URL http: / / www . utfit.org, together with continuously updated results of the UT analysis.

In the analysis within the Standard Model (SM), we have recently included in $\varepsilon_{K}$ the contributions of $\xi$ and $\phi_{\varepsilon} \neq \pi / 4$ which, as pointed out in [7, 8], decrease the SM prediction for $\varepsilon_{K}$ by $\sim 6 \%$.

The CKM matrix parameters are currently overconstrained and $\bar{\rho}$ and $\bar{\eta}$ are accurately determined: $\bar{\rho}=0.132 \pm 0.020, \bar{\eta}=0.358 \pm 0.012$ [9].

The consistency of the picture and the overconstraining is tested using compatibility plots that are comparing two different p.d.f.'s, the one obtained from the UT fit without using the constraint being tested and the other from the direct measurement. Their compatibility is evaluated by constructing the p.d.f. of their difference and by estimating the distance of its most probable value from zero in units of standard deviations. The latter is done by integrating this p.d.f. between zero and the most probable value and converting it into the equivalent number of standard deviations for a Gaussian distribution. The number of standard deviations between the measured value and the predicted value is plotted as a function of the measured value and its error. The compatibility can be then directly estimated on the plot, for any central value and error of the direct measurement. Fig. 2 shows few compatibility plots related to some key constraints. We can see how $\alpha$ and $\gamma$ show very good agreement with the rest of the fit, while $\sin 2 \beta, V_{c b}$ and $\varepsilon_{K}$ present some effects of 


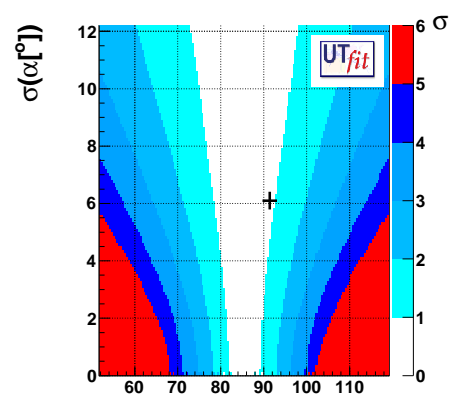

$\alpha\left[^{0}\right]$

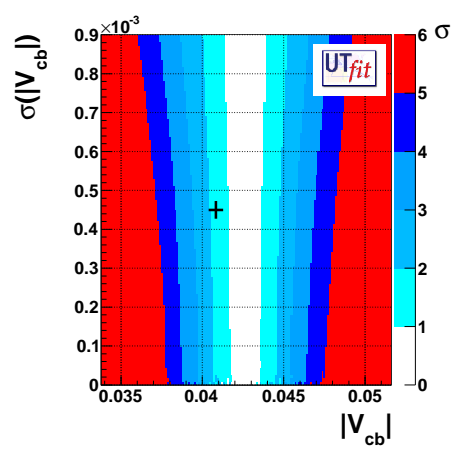

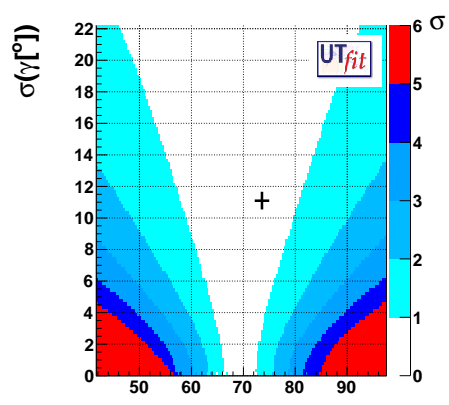

$\gamma\left[{ }^{0}\right]$

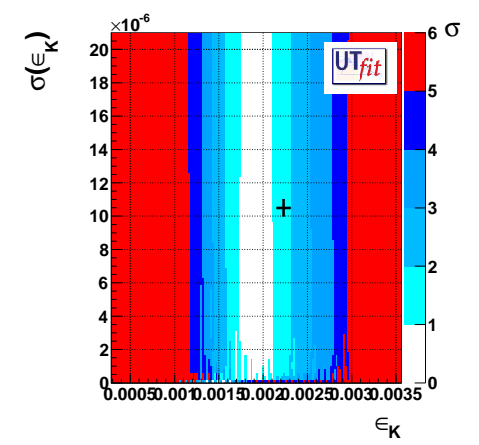

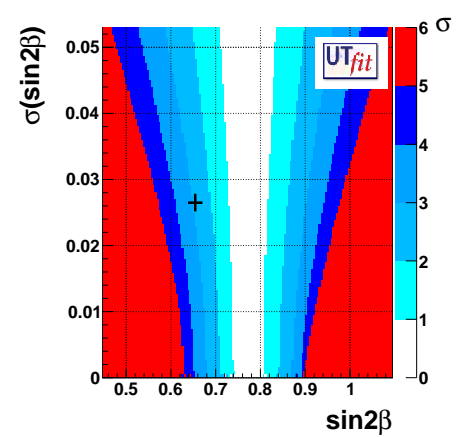

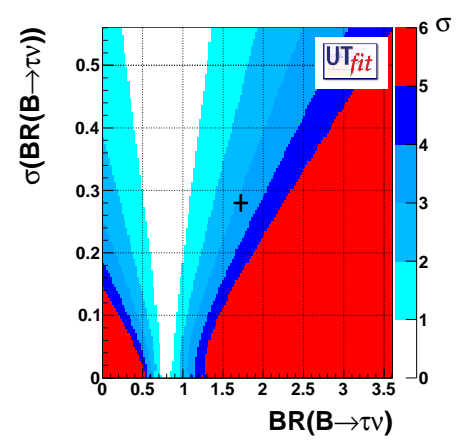

Figure 2: The compatibility between the direct and indirect determinations as a function of the measured values and errors. The compatibility regions from $1 \sigma$ to $6 \sigma$ are displayed. The crosses display the position (value/error) of the measurements. From left to right: top row shows $\alpha, \gamma$ and $\sin 2 \beta$. Bottom row shows $V_{c b}, \varepsilon_{K}$ and $B \rightarrow \tau v$.

disagreement.

We can say however that the UT analysis has established that the CKM matrix is the dominant source of flavour mixing and CP-violation and that New Physics (NP) effects can at most represent a small correction to this picture. The present tensions arise primarily from the new contributions in $\varepsilon_{K}$ affecting the constraints provided by the experimental measurements of $\varepsilon_{K}$ and $\sin 2 \beta$ (see fig. 1). A second, currently minor, source of tension comes from the $\left|V_{u b}\right|$ and $\left|V_{c b}\right|$ measurements and their internal tension between the exclusive and inclusive methods [10, 11].

As a consequence of these disagreements, the indirect determination of $\sin 2 \beta$ turns out to be larger than the experimental value by $\sim 2.6 \sigma .{ }^{1}$ In this regard, we observe that the new unquenched results [ $\llbracket$ for the bag-parameter $B_{K}$ tend to lie below older quenched results, thus enhancing the $\varepsilon_{K^{-}}-\sin 2 \beta$ tension.

From the full fit and keeping in mind these tensions, we can obtain useful SM predictions for other observables: for example, we have shown [13] how to use the UT fit to obtain the most accurate prediction of $\operatorname{BR}(B \rightarrow \tau \nu)$ in the $\mathrm{SM}$, thanks to a better determination of $\left|V_{u b}\right|$ and $f_{B}$. Within the SM, the UT fit prediction for $\mathrm{BR}(B \rightarrow \tau \nu)$ is found to deviate from the experimental measurement [3] by $\sim 3.2 \sigma$. Even allowing for NP effects in $\Delta F=2$ processes, while assuming negligible

\footnotetext{
${ }^{1}$ For an alternative indirect determination of $\sin 2 \beta$ which does not rely and is thus free from the hadronic uncertainty in $\left|V_{u b}\right|$, see ref. [12].
} 
NP contributions to the $B \rightarrow \tau \nu$ decay amplitude, a $\sim 2.2 \sigma$ deviation from the experimental value is found.

\section{Beyond the Standard Model: Unitarity Triangle Analysis in presence of New Physics}

We perform a full analysis of the UT with all the constraints studied for the classic SM UT analysis, but reinterpreting the experimental observables including possible model-independent NP contributions. Some other constraints are also added in order to extract more information on the NP parameters: these are detailed below.

This analysis consists first in generalising the relations among the experimental observables and the elements of the CKM matrix, introducing effective model-independent parameters that quantify the deviation of the experimental results from the SM expectations.

The possible NP effects considered in the analysis are those entering neutral meson mixing. Thanks to recent experimental developments, in fact, these $\Delta F=2$ processes turn out to provide stringent constraints on possible NP contributions.

The contribution of NP to $\Delta F=2$ transitions can be parameterised in a model-independent way as the ratio of the full $(\mathrm{SM}+\mathrm{NP})$ amplitude to the $\mathrm{SM}$ one. In this way, a complex effective parameter is introduced and it is defined as

$$
C_{B_{q}} e^{2 i \phi_{B_{q}}}=\frac{\left\langle B_{q}\left|H_{\mathrm{eff}}^{\mathrm{full}}\right| \bar{B}_{q}\right\rangle}{\left\langle B_{q}\left|H_{\mathrm{eff}}^{\mathrm{SM}}\right| \bar{B}_{q}\right\rangle}=\frac{A_{q}^{\mathrm{SM}} e^{2 i \phi_{q}^{\mathrm{SM}}}+A_{q}^{\mathrm{NP}} e^{2 i\left(\phi_{q}^{\mathrm{SM}}+\phi_{q}^{\mathrm{NP}}\right)}}{A_{q}^{\mathrm{SM}} e^{2 i \phi_{q}^{\mathrm{SM}}}},
$$

being $H_{e f f}^{S M}$ the SM $\Delta F=2$ effective Hamiltonian and $H_{\text {eff }}^{f u l l}$ its extension in a general NP model. The subscript $q$ represents $d$ or $s$, and by definition $C_{B_{d, s}}=1$ and $\phi_{B_{d, s}}=0$ within the SM. We also define an alternative NP parameterisation with an explicit NP phase $\phi_{q}^{\mathrm{NP}}$.

All the mixing observables are then expressed as a function of these parameters and the SM ones (see refs. [14, 15, 16] for details). For example, the mass differences and the CP asymmetries are related to the SM counterparts by:

$$
\Delta m_{q}=C_{B_{q}} \times \Delta m_{q}^{S M}, \beta_{q}=\beta_{q}^{S M}+\phi_{B_{q}}
$$

where in case of the $B_{s}$ system, the angle $\beta_{s}$ is defined as $\beta_{s}=\arg \left(-\left(V_{t s} V_{t b}^{*}\right) /\left(V_{c s} V_{c b}^{*}\right)\right)$ and it equals $0.018 \pm 0.001$ in the SM. ${ }^{2}$

In a similar way, for the $K-\bar{K}$ system, one can write

$$
C_{\varepsilon_{K}}=\frac{\operatorname{Im}\left[\left\langle K\left|H_{e f f}^{f u l l}\right| \bar{K}\right\rangle\right]}{\operatorname{Im}\left[\left\langle K\left|H_{\text {eff }}^{S M}\right| \bar{K}\right\rangle\right]}, \quad C_{\Delta m_{K}}=\frac{\operatorname{Re}\left[\left\langle K\left|H_{\text {eff }}^{\text {full }}\right| \bar{K}\right\rangle\right]}{\operatorname{Re}\left[\left\langle K\left|H_{\text {eff }}^{S M}\right| \bar{K}\right\rangle\right]},
$$

with $C_{\varepsilon_{K}}=C_{\Delta m_{K}}=1$ within the SM.

In $\varepsilon_{K}$ we have taken into account the effect of $\phi_{\varepsilon} \neq \pi / 4$, while the $\xi$ contribution, which beyond minimal flavour violation (MFV) [17, 18] is affected by a large uncertainty [19], is not included.

\footnotetext{
${ }^{2}$ We are using the usual CKM phase convention in which $V_{c s} V_{c b}^{*}$ is real to a very good approximation.
} 

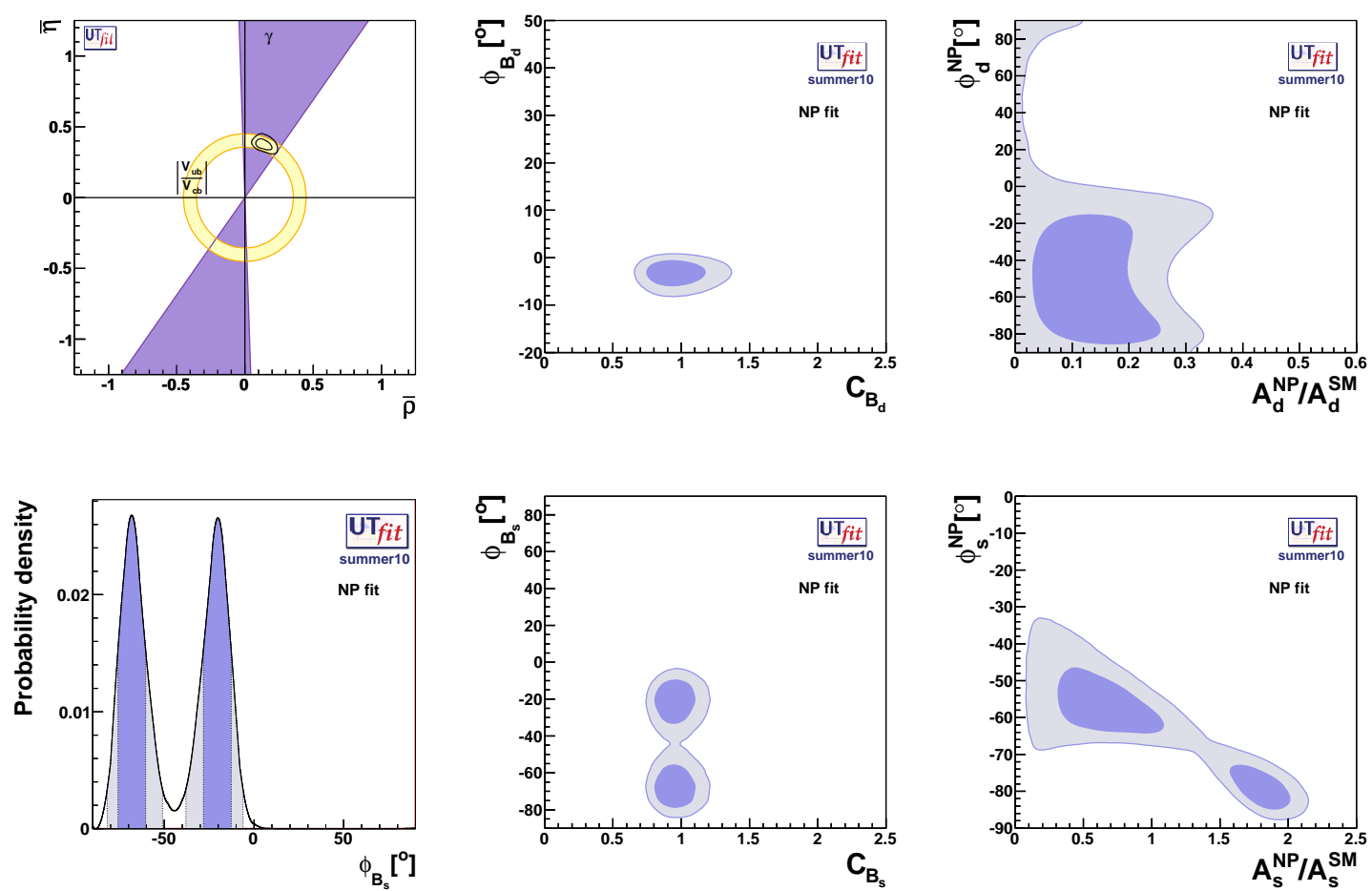

Figure 3: Top-left plot: determination of $\bar{\rho}$ and $\bar{\eta}$ from all the constraints. $68 \%$ and $95 \%$ total probability black contours are shown, together with $95 \%$ probability regions from the tree-only constraints. Remaining plots from left to right and from top to bottom: $68 \%$ (dark) and 95\% (light) probability regions in the $\phi_{B_{d}}-$ $C_{B_{d}}$ plane, $A_{d}^{N P} / A_{d}^{S M}-\phi_{d}^{N P}$ plane, 1-dimensional p.d.f's for $\phi_{B_{s}}, \phi_{B_{s}}-C_{B_{s}}$ plane and $A_{s}^{N P} / A_{s}^{S M}-\phi_{s}^{N P}$ plane.

As pointed out before, we add the following experimental inputs that are specifically thought to extract information on the NP parameters in the $B_{s}$ system: the semileptonic asymmetry in $B_{s}$ decays $A_{\mathrm{SL}}^{s}$ [3], the di-muon charge asymmetry $A_{\mathrm{SL}}^{\mu \mu}\left[20\right.$, the measurement of the $B_{s}$ lifetime from flavour-specific final states [3], the two-dimensional likelihood ratio for $\Delta \Gamma_{s}$ and $\phi_{s}=2\left(\beta_{s}-\right.$ $\phi_{B_{s}}$ ) from the time-dependent tagged angular analysis of $B_{s} \rightarrow J / \psi \phi$ decays. Regarding the latter constraint, here we are updating the $\mathbf{U} \mathbf{f}$ it analysis of ref. [21] by using the CDF and D0 results given as a combined two-dimensional likelihood without assumptions on the strong phases [22].

From the full NP analysis, the combined fit of all the experimental observables selects a region of the $(\bar{\rho}, \bar{\eta})$ plane $(\bar{\rho}=0.135 \pm 0.040, \bar{\eta}=0.374 \pm 0.026)$ which is consistent with the results of the SM analysis (see top-left plot in fig. 3 ). Together with the CKM parameters, we can also constrain the effective NP contributions in the three sectors.

For $K-\bar{K}$ mixing, the NP parameters are found in agreement with the SM expectations. In the $B_{d}$ system, the mixing phase $\phi_{B_{d}}$ is found $\simeq 1.8 \sigma$ away from the SM expectation, reflecting the slight tension between the direct measurement of $\sin 2 \beta$ and its indirect determination from the other UT constraints.

The $B_{s}$-meson sector, where the tiny SM mixing phase could be highly sensitive to a NP contribution, represents a privileged environment to search for NP. We find $\phi_{B_{s}}=(-68 \pm 8)^{\circ} \mathrm{U}$ $(-20 \pm 8)^{\circ}$, which is $3.1 \sigma$ away from the SM expectation $\phi_{B_{s}}=0$ (see bottom plots in fig. 3 ). 

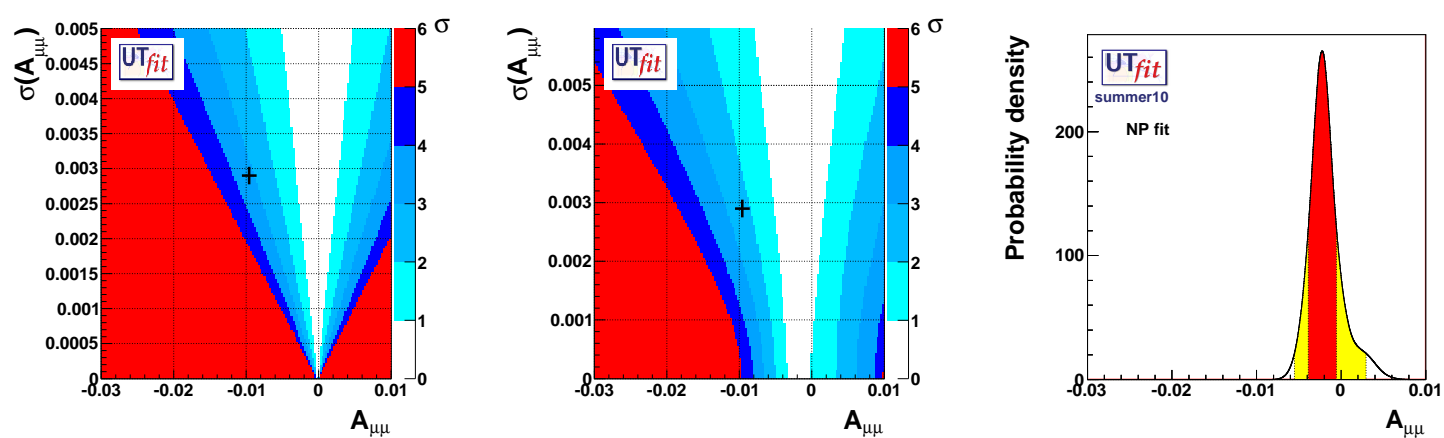

Figure 4: The compatibility between the direct and indirect determinations of the di-muon asymmetry in the SM fit (left) and in the NP fit (centre). The compatibility regions from $1 \sigma$ to $6 \sigma$ are displayed. The crosses display the position (value/error) of the measurement. Right plot: 1D distribution for $A_{\mu \mu}$ from the NP analysis without using the di-muon asymmetry as constraint. This represents the prediction on $A_{\mu \mu}$ taking into account generic NP contributions: we get the value $-0.0022 \pm 0.0017$.

A deviation of more than $2 \sigma$ is found also by the Heavy Flavour Averaging Group (HFAG) [3] $(2.2 \sigma)$ and by CKMfitter [23] $(2.5 \sigma)$, by combining the Tevatron results with some differences in the statistical approach.

At this conference CDF collaboration has presented the update of the time-dependent tagged angular analysis of $B_{s} \rightarrow J / \psi \phi$ decays with $5.2 \mathrm{fb}^{-1}$ of data [24]: this updated result gives a $\sin 2 \beta_{s}$ value compatible with the SM at better than $1 \sigma$ level. As a consequence of this, once the CDF collaboration makes the new likelihood publicly available, the NP $\phi_{B_{s}}$ phase in our NP analysis should show a smaller departure from the SM expectation.

However, we note that this kind of NP signal would be not only a signal of physics beyond the SM but more in general beyond MFV, since a value of $\phi_{B_{s}}$ different from zero can only be an effect of a new source of flavour violation different from the Yukawa couplings.

On the other side, the new D0 measurement of the di-muon asymmetry points to large $\beta_{s}$, but also to large $\Delta \Gamma_{s}$ requiring a non-standard $\Gamma_{12}$ for which our NP analysis does not account for. Fig. 4 show the compatibility plot for the di-muon asymmetry in both the SM and NP analyses: we see that allowing for NP does not accommodate the current value going from the $3.2 \sigma$ to a $2.2 \sigma$ disagreement. If this result is confirmed, this can lead towards two possible scenarios both quite difficult to accommodate with the rest of the flavour data: huge (tree-level-like) NP contributions in $\Gamma_{12}$ or a bad failure of the OPE in $\Gamma_{12}[25]$.

\section{References}

[1] M. Ciuchini et al., 2000 CKM-triangle analysis: A critical review with updated experimental inputs and theoretical parameters, JHEP 0107 (2001) 013 [hep-ph/0012308].

[2] M. Bona et al. [UTfit Collaboration], The 2004 UTfit Collaboration report on the status of the unitarity triangle in the standard model, JHEP 0507 (2005) 028 [hep-ph/0501199].

[3] The Heavy Flavour Averaging Group (HFAG), http://www.slac.stanford.edu/xorg/hfag/.

[4] V. Lubicz, Kaon physics from lattice QCD, PoS LAT2009 (2009) 013 [arXiv:1004.3473 [hep-lat]]. 
[5] J. Laiho, E. Lunghi and R. S. Van de Water, Lattice QCD inputs to the CKM unitarity triangle analysis, Phys. Rev. D 81 (2010) 034503 [arXiv:0910.2928 [hep-ph]].

[6] V. Lubicz and C. Tarantino, Flavour physics and Lattice QCD: averages of lattice inputs for the Unitarity Triangle Analysis, Nuovo Cim. 123B (2008) 674 [arXiv:0807.4605 [hep-lat]].

[7] A. J. Buras and D. Guadagnoli, Correlations among new CP violating effects in $\Delta F=2$ observables, Phys. Rev. D 78 (2008) 033005 [arXiv:0805.3887 [hep-ph]].

[8] A. J. Buras, D. Guadagnoli and G. Isidori, On $\varepsilon_{K}$ beyond lowest order in the Operator Product Expansion, Phys. Lett. B 688 (2010) 309 [arXiv:1002.3612 [hep-ph]].

[9] The UTfit Collaboration, http://www.utfit.org/.

[10] M. Bona et al. [UTfit Collaboration], The unitarity triangle fit in the standard model and hadronic parameters from lattice $Q C D:$ A reappraisal after the measurements of $\Delta m_{s}$ and $B R\left(B \rightarrow \tau \nu_{\tau}\right)$, JHEP 0610 (2006) 081 [arXiv:hep-ph/0606167].

[11] R. Kowalewski, Measurement of $\left|V_{c b}\right|$ and $\left|V_{u b}\right|$, talk and proceeding from this conference.

[12] E. Lunghi and A. Soni, Possible Indications of New Physics in $B_{d}$-mixing and in $\sin (2 \beta)$ Determinations, Phys. Lett. B 666 (2008) 162 [arXiv:0803.4340 [hep-ph]].

[13] M. Bona et al. [UTfit Collaboration], An Improved Standard Model Prediction Of BR $(B \rightarrow \tau v)$ And Its Implications For New Physics, arXiv:0908.3470 [hep-ph].

[14] M. Bona et al. [UTfit Collaboration], The UTfit collaboration report on the status of the unitarity triangle beyond the standard model. I: Model-independent analysis and minimal flavour violation, JHEP 0603 (2006) 080 [hep-ph/0509219].

[15] M. Bona et al. [UTfit Collaboration], The UTfit collaboration report on the unitarity triangle beyond the standard model: Spring 2006, Phys. Rev. Lett. 97 (2006) 151803 [hep-ph/0605213].

[16] M. Bona et al. [UTfit Collaboration], Model-independent constraints on $\Delta F=2$ operators and the scale of New Physics, JHEP 0803 (2008) 049 [0707.0636 [hep-ph]].

[17] G. D'Ambrosio et al., Minimal flavour violation: An effective field theory approach, Nucl. Phys. B 645 (2002) 155 [hep-ph/0207036].

[18] A. J. Buras et al., Universal unitarity triangle and physics beyond the standard model, Phys. Lett. B 500 (2001) 161 [hep-ph/0007085].

[19] A. J. Buras and D. Guadagnoli, On the consistency between the observed amount of CP violation in the $K$ - and $B_{d}$-systems within minimal flavor violation, Phys. Rev. D 79 (2009) 053010 [arXiv:0901.2056 [hep-ph]].

[20] V. M. Abazov et al. [D0 Collaboration], Evidence for an anomalous like-sign dimuon charge asymmetry, Phys. Rev. D 82, 032001 (2010) [arXiv:1005.2757 [hep-ex]].

[21] M. Bona et al. [UTfit Collaboration], First Evidence of New Physics in $b \leftrightarrow s$ Transitions, 0803.0659 [hep-ph].

[22] The Tevatron B Working Group, http://tevbwg.fnal.gov/: Summer $2009 \sin 2 \beta_{\text {s }}$ combination http://tevbwg.fnal.gov/results/Summer2009_betas/

[23] O. Deschamps, CKM global fit and constraints on New Physics in the B meson mixing, arXiv:0810.3139 [hep-ph]. 
[24] talk at this conference by Louise Oakes, Measurement of $\beta_{s}$ at $C D F$, public result web-page at URL http://www-cdf.fnal.gov/physics/new/bottom/100513.blessed-BsJpsiPhi_5.2fb/

[25] A. Dighe, A. Kundu and S. Nandi, Enhanced $B_{s}-\bar{B}_{s}$ lifetime difference and anomalous like-sign dimuon charge asymmetry from new physics in $B_{S} \rightarrow \tau^{+} \tau^{-}$, Phys. Rev. D 82 (2010) 031502 [arXiv:1005.4051 [hep-ph]];

C. W. Bauer and N. D. Dunn, Comment on new physics contributions to $\Gamma_{12}^{s}$, arXiv:1006.1629 [hep-ph];

Z. Ligeti, M. Papucci, G. Perez and J. Zupan, Implications of the dimuon CP asymmetry in $B_{d, s}$ decays, arXiv:1006.0432 [hep-ph];

B. A. Dobrescu, P. J. Fox and A. Martin, CP violation in $B_{s}$ mixing from heavy Higgs exchange, Phys. Rev. Lett. 105 (2010) 041801 [arXiv:1005.4238 [hep-ph]];

K. Blum, Y. Hochberg and Y. Nir, Implications of large dimuon CP asymmetry in $B_{d, s}$ decays on minimal flavor violation with low tan beta, JHEP 1009 (2010) 035 [arXiv:1007.1872 [hep-ph]]; 